\title{
A consensus statement on empiric therapy for suspected gram-positive infections in surgical patients
}

\author{
Joseph S. Solomkin, M.D. ${ }^{\mathrm{a}, \mathrm{b}, *}$, H. Stephen Bjornson, M.D., Ph.D. , \\ Miguel Cainzos, M.D., Ph.D. ${ }^{c}$, E. Patchen Dellinger, M.D. ${ }^{\mathrm{d}}$, Lorenzo Dominioni, M.D. ${ }^{\mathrm{e}}$, \\ Robert Eidus, M.D., M.B.A. ${ }^{\mathrm{f}}$, Eugen Faist, M.D. ${ }^{\mathrm{g}}$, David Leaper, M.D. ${ }^{\mathrm{h}}$, \\ James T. Lee, M.D., Ph.D. ${ }^{\mathrm{i}}$, Pamela A. Lipsett, M.D. ${ }^{\mathrm{j}}$, Lena Napolitano, M.D. ${ }^{\mathrm{k}}$, \\ Carl L. Nelson, M.D. ${ }^{1}$, Robert G. Sawyer, M.D. ${ }^{\mathrm{m}}$, John Weigelt, D.V.M., M.D. ${ }^{n}$, \\ Samuel Eric Wilson, M.D. ${ }^{\circ}$ \\ ${ }^{a}$ Division of Trauma and Critical Care, University of Cincinnati College of Medicine, Cincinnati, OH, USA \\ ${ }^{\mathrm{b}}$ Department of Surgery, Division of Trauma/Critical Care, University of Cincinnati College of Medicine, 231 Albert B. Sabin Way, Cincinnati, OH \\ 45267-0558, USA \\ ${ }^{\mathrm{c}}$ Medical School of the University of Santiago de Compostela and Hospital General de Galicia, Santiago de Compostela, Spain \\ ${ }^{\mathrm{d}}$ Division of General Surgery, University of Washington Medical Center, Seattle, WA, USA \\ ${ }^{\mathrm{e}}$ Center for Thoracic Surgery, University of Insubria, Ospedale di Circolo di Varese, Varese, Italy \\ ${ }^{\mathrm{f}}$ Einstein Practice Plan, Philadelphia, PA, USA \\ ${ }^{\mathrm{g}}$ University of Munich, Klinikum Grosshadern, Munich, Germany

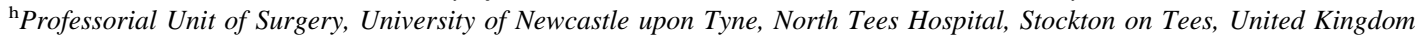 \\ ${ }^{i}$ Specialty Care Patient Service Line, Department of Veterans Affairs Medical Center, Minneapolis, MN, USA \\ ${ }^{\mathrm{j} D e p a r t m e n t}$ of Surgery, The Johns Hopkins University School of Medicine and Surgical Intensive Care Units, The Johns Hopkins Hospital, Baltimore, MD, USA \\ ${ }^{\mathrm{k}}$ Surgical Intensive Care Unit, Veterans Administration Hospital, Baltimore, MD, USA \\ ${ }^{1}$ University of Arkansas, Little Rock, AK, USA \\ ${ }^{\mathrm{m}}$ University of Virginia Health Sciences Center and University Hospital West, Charlottesville, VA, USA \\ ${ }^{\mathrm{n}}$ Department of Surgery and the Division of Trauma and Critical Care, Medical College of Wisconsin, Milwaukee, WI, USA \\ ${ }^{\circ}$ Department of Surgery, University of California Irvine College of Medicine, Orange, CA, USA
}

Manuscript received August 19, 2002; revised manuscript March 3, 2003

\begin{abstract}
Background: Multidrug resistance among gram-positive pathogens in tertiary and other care centers is common. A systematic decision pathway to help select empiric antibiotic therapy for suspected gram-positive postsurgical infections is presented.

Data sources: A Medline search with regard to empiric antibiotic therapy was performed and assessed by the 15-member expert panel. Two separate panel meetings were convened and followed by a writing, editorial, and review process.

Conclusions: The main goal of empiric treatment in postsurgical patients with suspected gram-positive infections is to improve clinical status. Empiric therapy should be initiated at the earliest sign of infection in all critically ill patients. The choice of therapy should flow from $\beta$-lactams to vancomycin to parenteral linezolid or quinupristin-dalfopristin. In patients likely to be discharged, oral linezolid is an option. Antibiotic resistance is an important issue, and in developing treatment algorithms for reduction of resistance, the utility of these new antibiotics may be extended and reduce morbidity and mortality. (C) 2004 Excerpta Medica, Inc. All rights reserved.
\end{abstract}

Keywords: Methicillin-resistant staphylococci; Gram-positive infection; Antibiotic resistance; Empiric treatment; Postsurgical infections; Linezolid; $\beta$ Lactams; Vancomycin; Quinupristin/dalfopristin

Gram-positive organisms have emerged as prominent pathogens in nosocomial and postoperative infections. Coagulase-negative staphylococci (CoNS), Staphylococcus

\footnotetext{
* Corresponding author. Tel.: +1-513-558-4427; fax: +1-513-558-3136.

E-mail address: joseph.solomkin@uc.edu
}

aureus, and enterococci now account for $50 \%$ to $60 \%$ of the cases of nosocomial bacteremia (Fig. 1) [1,2]. These data reflect the increasing incidence of infections caused by gram-positive pathogens in Europe and North America since the mid-1970s [3].

Multidrug resistance among gram-positive pathogens has 


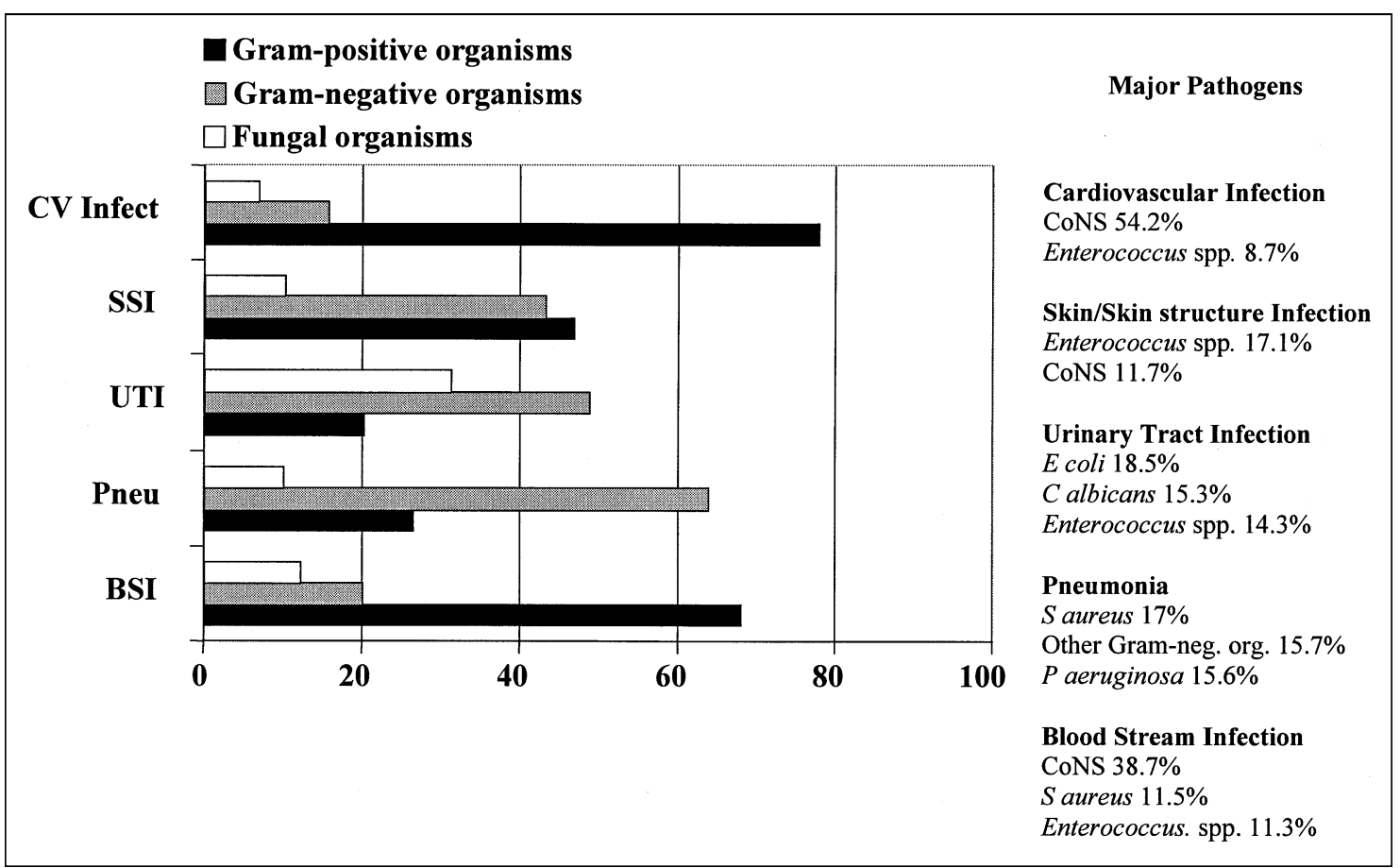

Fig. 1. Percentage of pathogens reported at selected sites of nosocomial infection for patients in combined medical-surgical intensive care units, National Nosocomial Infection Surveillance System, 1992-1998 [2]. (CV infect $=$ cardiovascular infection; SSI $=$ surgical-site infection; UTI = urinary tract infection; pneu = pneumonia; BSI = bloodstream infection; CoNS $=$ coagulase-negative staphylococci; enteroc $=$ enterococci; gram-neg org $=$ gram-negative organism.)

increased to the point of being a common finding in many centers. The development of such resistance is an important clinical problem because of the potential risk of providing inadequate empiric therapy and because more complex antiinfective regimens may be required to control these infections. Success rates in the management of gram-positive infections in postsurgical patients are improved by early therapy with agents active against the organisms subsequently identified by appropriate culture and susceptibility testing. This is supported by clinical studies in patients with ventilator-associated pneumonia, peritonitis, bacteremia, and meningitis, where provision of inadequate therapy is closely correlated with adverse patient outcomes, including increased rates of hospital mortality [4-8].

There are several reasons for the appearance of $\beta$-lactam resistant gram-positive organisms in hospital settings. The wide use of agents with high potency against gram-negative organisms but considerably less activity against gram-positive pathogens has contributed significantly to the emergence of resistance. Such reduced activity allows for prolonged exposure of initially susceptible organisms to suboptimal concentrations of $\beta$-lactams, a situation facilitating selection of resistant strains.

Methicillin resistance refers collectively to $\beta$-lactam resistance and may be conferred either by the production of $\beta$-lactamases or through production of low-affinity penicillin-binding proteins (PBPs), inhibiting antibiotic interaction with the bacterial cell membrane and subsequent bactericidal activity. All strains of methicillin-resistant $S$ aureus
(MRSA) produce such altered PBPs, such as PBP2 or PBP2a [9]. The production of PBPs is regulated by a cluster of chromosomal genes, making the expression of resistance a chromosomally-mediated phenomenon [10]. Most strains of MRSA are also resistant to multiple drugs, particularly to aminoglycosides.

\section{Antibiotic options in the treatment of resistant gram- positive cocci}

The glycopeptide vancomycin has been the antibiotic of choice for $\beta$-lactam resistant gram-positive organisms. However, the utility of this agent has diminished with the increasing incidence of vancomycin-resistant enterococci (VRE), the appearance of vancomycin-resistant $S$ aureus (VRSA) [11], and glycopeptide-intermediate or vancomycin-intermediate $S$ aureus (GISA/VISA) [12]. Current information on the incidence of vancomycin-resistant organisms may be obtained from www.cdc.gov. Additionally, heterogeneous resistance to vancomycin has been reported in MRSA isolates from nosocomial and surgical infections [13-15]; this may be an additional explanation for therapeutic failure with vancomycin in MRSA infections. Such developments suggest that empiric therapy with newer agents with different mechanisms of actions, such as the streptogramin combination, quinupristin/dalfopristin (Q/D), and the oxazolidinone, linezolid, will be important in the treatment of such infections. 


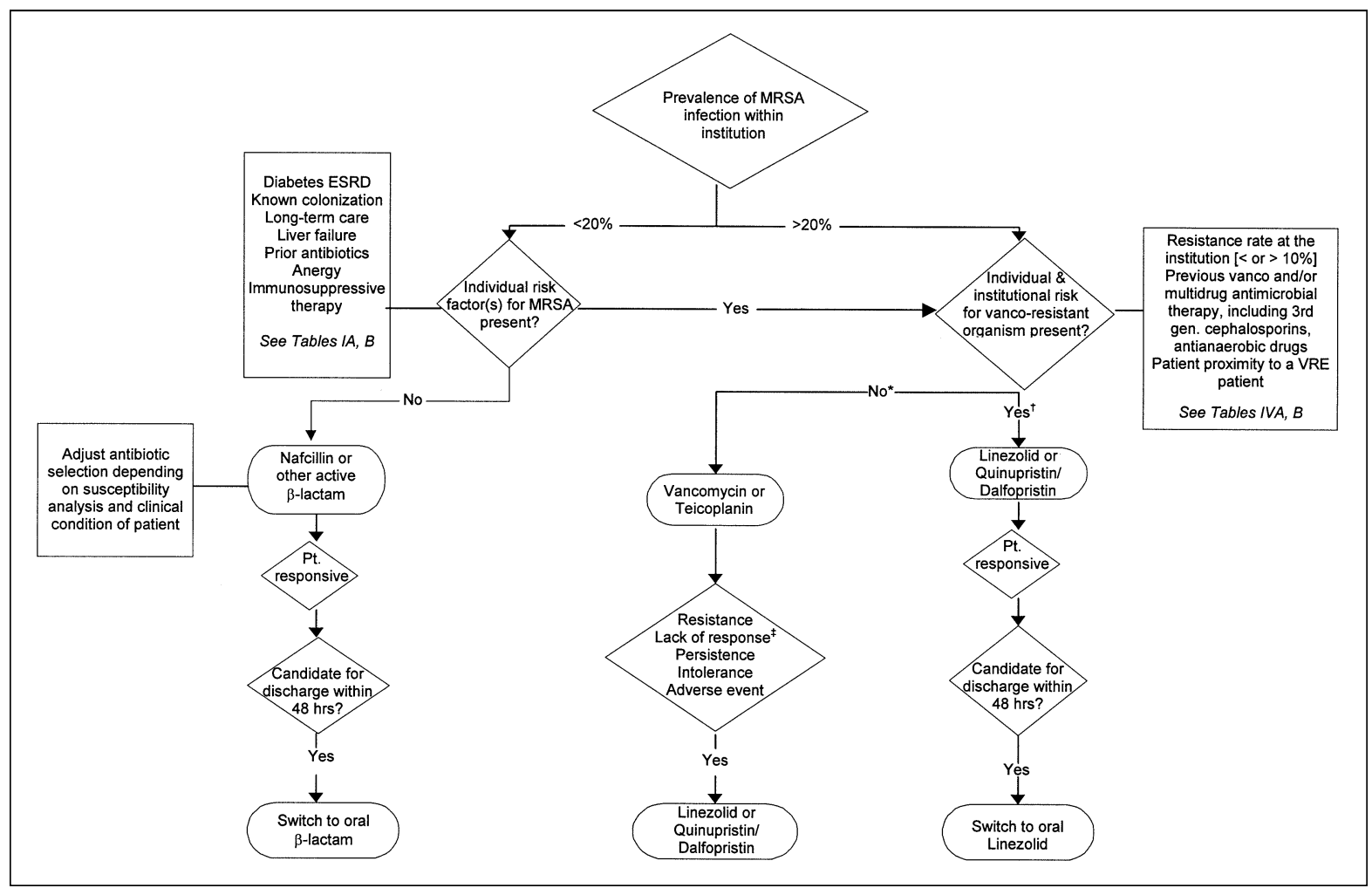

Fig. 2. The decision tree for treatment of a patient suspected of having a gram-positive infection. *Institutional risk for VRE $<10 \%$, no individual risk for VRE. †Institutional risk for VRE $>10 \%$ or individual risk for VRE present. $\$$ Lack of response assumes no drainable abscess or removable infected device. $($ MRSA = methicillin-resistant Staphylococcus aureus; ESRD = end-stage renal disease; vanco = vancomycin; gen. = generation; VRE = vancomycinresistant enterococci; $\mathrm{pt}=$ patient.)

However, there are important consequences of providing needlessly broad empiric therapy. Aside from the cost of combination regimens needed to cover the broad range of possible infecting microorganisms, there is risk of colonization with a resistant flora in the patient so treated [16]. In particular, restriction of vancomycin has been urged as a means of reducing the incidence of vancomycin resistance [17].

\section{The consensus panel approach}

The increasing frequency of multidrug resistant grampositive organisms in postsurgical infections and the availability of an expanding number of agents active against these organisms produce an array of decisions regarding the appropriate empiric management of suspected gram-positive infections for a particular patient. To define and address the decisions involved in the management of suspected or proven gram-positive infections, an expert panel was convened.

The goal of the panel was to develop a treatment algorithm based on available evidence as extracted from Medline searches. A preliminary survey of the literature indicated a dearth of randomized, controlled trials with regard to empiric antibiotic therapy in general and a lack of prospec- tive treatment data relating to specific antibiotics. While the panel recognized the importance of the development of evidence-based guidelines, it acknowledged the paucity of data in this area and, considering the critical public health issues in infectious disease and antibiotic resistance, decided to develop a treatment algorithm based on evidence from retrospective studies and reviews. Where published evidence was insufficient for clear definition, the collective experience of the 15-member panel was used to identify reasonable strategies for patient management through consensus. Two separate panel meetings were convened and followed by a writing and review process.

The purpose of this article is to present a systematic decision pathway to aid in the clinical selection of antibiotics as empiric therapy for suspected gram-positive postsurgical infections.

\section{The decision tree}

The decision tree describes the flow of events and management decisions that must be made once it has been determined to treat a patient for a suspected gram-positive infection. Fig. 2 is a flow chart presenting the sequence of events, while the text provides the criteria and evidence for each decision. The decision tree also identifies specific 
questions that should be asked and includes treatment options for possible answers.

Because of the wide variety of infections that may occur in a postsurgical patient, the elements of an appropriate work-up for each patient will not be detailed. It is important to note, however, that every effort should be made to culture suspected sources of infection. Appropriate techniques for obtaining samples have been described in guidelines for catheter-related infections [18], surgical site infections [19], and ventilator-associated pneumonia [20].

The costs of delaying therapy until results of culture and susceptibility testing are available for patients not experiencing life-threatening illnesses are less severe, and the physician may decide to continue observation or conduct further diagnostic evaluation. An important concern, however, remains the consequences of delayed treatment of $S$ aureus bacteremia $[8,21]$. There is abundant evidence that staphylococcal bacteremia causes a variety of infectious complications, including metastatic abscess formation and endocarditis [22]. Current evidence would support a 10 to 15 day treatment course for patients whose bacteremia and clinical illness clear within 3 days of catheter discontinuation and antibiotic therapy $[23,24]$.

Given the above data and understanding the need to avoid overexposure to antibiotics, the panel recommends that empiric therapy be instituted in patients with underlying illness who demonstrate evidence of infection or in whom the risk of waiting for a definitive culture result is greater than the risk of early intervention. In such patients, empiric antibiotic therapy should be instituted early and with an antimethicillin-resistant staphylococcal (MRS) agent. In patients in whom presumed infections are likely to include gram-negative bacteria or fungi, coverage for these pathogens should also be included.

\section{Institutional prevalence considerations}

Determining the threshold incidence of MRSA that warrants empiric antibiotic therapy for resistant organisms is a complex decision based upon a variety of factors. These factors include the prevalence rates of MRSA, GISA, and VRE at the specific hospital, the consequences of inadequate empiric therapy in the particular patient, the acquisition costs of potentially usable therapeutic agents, the differential efficacy of these agents, the risk of induction of resistance, and the general and patient-specific toxicities of each agent. It must be emphasized that any cut-off point is arbitrary and the physician's judgment and assessment of institutional factors and patient risk should always play a central role in decision-making.

There is currently no evidence to support any definitive cut-off point applicable to all patients. It is the consensus of the expert panel that prevalence rates for MRSA $>20 \%$ should be considered high, and the risk to the individual patient of treatment failure becomes substantial (Fig. 2). This issue is, however, rapidly becoming moot. The spread
Table 1

Risk factors

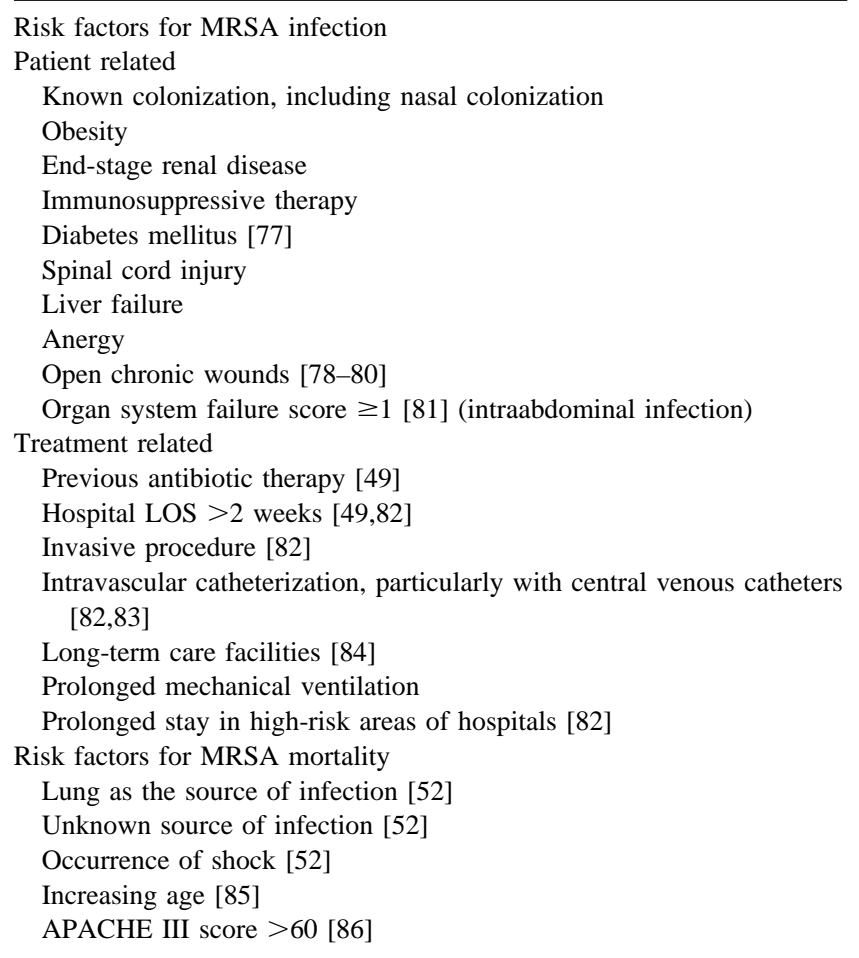

MRSA = methicillin-resistant Staphylococcus aureus; LOS = length of stay; ICU = intensive care unit; APACHE III = Acute Physiology and Chronic Health Evaluation III.

of methicillin resistance is truly remarkable. In $1981,5 \%$ of all $S$ aureus isolates from patients in large teaching hospitals were methicillin resistant. By 1991, this number had increased to $38 \%$ [25]. The latest NNIS data indicate that methicillin resistance is as high as 54\%, an increase of $40 \%$ in just 4 years. As the SCOPE (Surveillance and Control of Pathogens of Epidemiologic Importance) data indicate, there is both regional and intrainstitutional variation in the distribution of such resistant pathogens [1]. Rates are currently highest in the eastern United States. Within hospitals, it appears that the general surgical and cardiothoracic services have the highest proportions of MRSA (40\%). Thus, geographic and intrainstitutional considerations must be factored into any risk assessment.

\section{Individual risk-factor considerations}

When institutional prevalence rates of MRSA are low $(<20 \%)$, the choice of antibiotic therapy may be a synthetic penicillin such as nafcillin. The $\beta$-lactam antibiotics are the drugs of choice for staphylococcal infections [26]. However, when individual risk factors for MRSA infection are present, the choice of antibiotic should shift to vancomycin, $\mathrm{Q} / \mathrm{D}$, or linezolid. Table 1 provides a list of patient- and treatment-related factors that increase individual risk of MRSA infection and mortality and include factors such as 
diabetes, end-stage renal disease, prior antibiotic therapy, and prior immunosuppressive therapy.

In patients who are candidates for anti-MRS therapy, decisions have to be made regarding which antibiotic to use and the method and length of administration of such an agent. (Table 2 [available from author] provides a comparative profile of key anti-MRS agents and is available from the senior author on direct request.)

\section{Vancomycin}

Vancomycin is a glycopeptide antibiotic that exerts bactericidal activity via inhibition of bacterial cell wall biosynthesis. The only agent available until quite recently for $\beta$-lactam resistant gram-positive organisms, vancomycin, has had a remarkably long duration of use. However, its nephrotoxic side effects are a continuing concern [27]. Since it enhances the toxicity of aminoglycosides, it is probable that vancomycin injures renal tubular epithelial cells [28].

With the exception of the incidences of VRSA and VISA noted above, virtually all strains of $S$ aureus are susceptible to vancomycin. The vast majority of CoNS are also susceptible to vancomycin. Enterococci, however, are a different issue, and the increasing prevalence of VRE among postsurgical infections has become a major public health issue. Vancomycin is bactericidal for most gram-positive pathogens, with minimum inhibitory concentrations (MICs) in the range of 1 to $5 \mu \mathrm{g} / \mathrm{mL}$. For enterococci, vancomycin is only bacteriostatic.

\section{Quinupristin/dalfopristin}

Quinupristin/dalfopristin is a combination of two streptogramins that exert activity via inhibition of protein synthesis. Each component binds to the peptidyltransferase domain of the bacterial ribosome with differing affinity and actions [29]. The implication of this is that development of resistance by susceptible organisms would be uncommon. In practice, acquisition of resistance appears to occur at rates observed with other agents, including vancomycin and linezolid. Resistance may develop through decreased ribosomal binding, through inactivating enzymes, or through efflux mechanisms [30]. In vitro analysis of the spectrum of activity of Q/D has confirmed its relatively selective coverage of gram-positive aerobic bacteria.

Individually, each component of the streptogramin has bacteriostatic activity against staphylococci and streptococci, but together, the agents exhibit synergy leading to bactericidal activity. The combination drug, however, is bacteriostatic against Enterococcus faecium and has poor activity against Enterococcus faecalis. Despite a short halflife, an extended postantibiotic effect allows the agent to be dosed every 8 to 12 hours.

Quinupristin/dalfopristin inhibits the cytochrome P450 3A4 pathway, and caution is warranted with concomitant use of other medications eliminated via this pathway. Clin-
Table 3

Antimicrobial activities of vancomycin, linezolid, and quinupristindalfopristin against various bacterial strains [33]

\begin{tabular}{lcll}
\hline Organism & $\mathrm{MIC}_{90}$ & & \\
\cline { 2 - 4 } & $\begin{array}{l}\text { Vancomycin } \\
\text { (range)* }\end{array}$ & $\begin{array}{l}\text { Quinupristin/ } \\
\text { Dalfopristin } \\
\text { (range) }\end{array}$ & $\begin{array}{l}\text { Linezoid } \\
\text { (range) }\end{array}$ \\
\hline MSSA & $2.0(0.50-2.0)$ & $1.0(\mathrm{NA})$ & $2.0(1.0-2.0)$ \\
MRSA & $2.0(0.50-4.0)$ & $1.0(\mathrm{NA})$ & $2.0(0.25-4.0)$ \\
MSCoNS & $2.0(0.25-2.0)$ & $0.5(\mathrm{NA})$ & $1.0(0.25-2.0)$ \\
MRCoNS§ & $2.0(0.5-4.0)$ & 0.5 (NA) & $2.0(0.5-4.0)$ \\
VS E faecalis & $2.0(0.5-4.0)$ & NA & $2.0(0.5-2.0)$ \\
VR E faecalis & $1024.0(16.0-1024.0)$ & NA & $2.0(1.0-2.0)$ \\
VS E faecium & $2.0(\leq 0.01-2.0)$ & NA & $2.0(0.5-2.0)$ \\
VR E faecium & $512.0(16.0-1024.0)$ & 1.0 (NA) & $2.0(1.0-2.0)$ \\
\hline
\end{tabular}

* Isolate counts as follows: MSSA (31), MRSA (50), MSCoNS (28), MRCoNS (46), VS E faecalis (25), VR E faecalis (17), VS E faecium (27), and VR E faecium (38).

$\dagger$ Isolate counts as follows: MSSA (2,140), MRSA (1051), MSCoNS (940), MRCoNS (786), and VS E faecium (895).

$\ddagger$ Isolate counts as follows: MSSA (31), MRSA (50), MSCoNS (28), MRCoNS (46), VS E faecalis (25), VR E faecalis (17), VS E faecium (27), and VR E faecium (38).

$\S$ Includes 15 teicoplanin-resistant strains.

MIC $=$ minimum inhibitory concentration; MSSA $=$ methicillin-susceptible Staphylococcus aureus; MRSA = methicillin-resistant Staphylococcus aureus; MSCoNS = methicillin-susceptible coagulase-negative staphylococci; MRCoNS = methicillin-resistant coagulase-negative staphylococci; VS = vancomycin susceptible; VR = vancomycin resistant; NA $=$ not available.

ical trials in patients with vancomycin-resistant $E$ faecium infections have documented efficacy [31]. The results of comparative clinical trials suggest that $\mathrm{Q} / \mathrm{D}$ has similar efficacy to that of commonly used antibiotics, including cefazolin, oxacillin, and vancomycin, in patients with skin and skin-structure infections or nosocomial pneumonia. Arthralgia, myalgia, nausea, diarrhea, vomiting, and rash are the most frequently reported systemic adverse events [31].

\section{Linezolid}

Linezolid is a synthetic antibiotic that inhibits bacterial protein translation at the initiation phase of protein synthesis [32]. All linezolid active against MRSA and VRE (Table 3) [33-37]. For vancomycin-susceptible enterococci, staphylococci, and streptococci, the activity of linezolid appears comparable to that of vancomycin [34]. Linezolid is rapidly and completely absorbed after oral administration and reaches peak plasma concentrations within 1 to 2 hours. At steady state, minimum plasma concentrations following standard dosing have been found to be above $\mathrm{MIC}_{90}$ concentrations for susceptible pathogens.

Linezolid has been found clinically efficacious in the treatment of skin and skin structure, nosocomial pneumonia, and bloodstream infections due to VRE $[38,39]$. It is generally well tolerated, with gastrointestinal disturbances being the most commonly occurring adverse event. Linezolid can suppress the bone marrow in a time-dependent manner 
Table 4

Institutional and individual risk factors for VRE

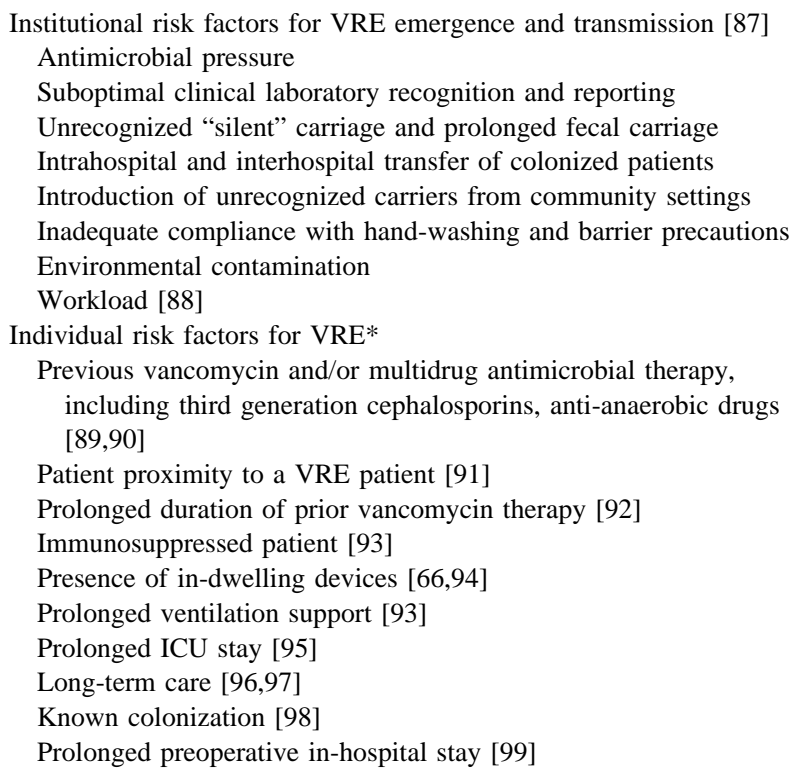

* Factor in potential adverse outcome to the individual patient of delayed therapy.

VRE = vancomycin-resistant enterococci; ICU = intensive care unit.

[40]. Thrombocytopenia is the most common manifestation of this phenomenon and occurred in approximately $2 \%$ of patients in clinical trials. Discontinuation of linezolid results in restoration of platelet counts to pretreatment levels in patients for whom outcomes are known. As a precaution, complete blood counts should be performed weekly in patients who require greater than 2 weeks of therapy, who are at increased risk for bleeding, who have preexisting thrombocytopenia, or who receive other medications that may cause thrombocytopenia.

\section{Selection of therapy for presumed methicillin-resistant staphylococci}

In patients for whom an anti-MRS agent is indicated, empiric treatment may be initiated with vancomycin, linezolid, or Q/D. Both institutional and individual risk factors should be weighed in making the decision. Vancomycin or teicoplanin (in Europe) may be the antibiotic of choice when individual risks and institutional risks for VRE are low $(<10 \%)$.

\section{Selection of therapy when risk of vancomycin resistance is present}

Table 4 provides a list of risk factors associated with the development of VRE. Vancomycin should not be prescribed when institutional risks for VRE infections are high $(>10 \%)$ or in cases in which an individual's risk for VRE acquisition is high. As with MRSA, there are no data that indicate the cut-off points for vancomycin therapy. However, the same variables that define individual patient thresholds for the assumption of resistance apply.

\section{Problems associated with vancomycin therapy}

Recent clinical studies have shown less than optimal results in the treatment of bacteremia and endocarditis with vancomycin $[7,41,42]$. Numerous reports have also documented that MRSA bacteremia may persist during vancomycin therapy despite resolution of fever and may lead to significantly delayed complications [43]. Another recent study has also demonstrated that MRSA colonization of a silastic rubber surface can occur even in the presence of prophylactic vancomycin or rifampin [44].

Also of concern is the significant mortality rate, approximately $50 \%$, in patients treated with vancomycin for MRSA pneumonia [45]. In contrast, in patients receiving $\beta$-lactams for pneumonia caused by methicillin-susceptible $S$ aureus (MSSA), survival is the rule [46]. Because of difficulties in clearly defining nosocomial pneumonia, particularly in ventilated patients, the outcome of bacteremia in patients with pneumonia is of interest. A recent small observational (without randomization) study reported high mortality rates in patients treated with vancomycin for bacteremic nosocomial pneumonia caused either by MRSA or MSSA [47]. In the group of patients with MSSA pneumonia, infection-associated mortality was found to be significantly higher among vancomycintreated patients $(47 \%)$ than among those treated with cloxacillin $(0 \%, P<0.01)$. Multivariate analysis showed a relationship between mortality and the following variables: septic shock (odds ratio $[\mathrm{OR}]=61$ ), vancomycin treatment $(\mathrm{OR}=14)$, and respiratory distress $(\mathrm{OR}=8)$. Similar concerns were articulated by Moise et al [48]. Lack of lung tissue penetration may be the underlying cause of treatment failure with vancomycin in pneumonia.

Many smaller studies showing no difference between mortality caused by MSSA and that caused by MRSA [49,50]. However, in a recent meta-analysis of 31 cohort studies published between 1980 and 2000, MRSA bacteremia was shown to be clearly associated with a higher mortality rate than MSSA bacteremia [51]. This difference persisted when subanalyses were done for length of stay (LOS) and severity of illness. Other studies have documented higher mortality rates and resource utilization with MRSA infections compared with MSSA infections [7,52-54]. These studies only serve to highlight the critical nature and multiple consequences of the problem of antibiotic resistance.

\section{Tolerance and heteroresistance as potential mechanisms for treatment failure}

Treatment failures with vancomycin have prompted studies investigating the phenomenon of tolerance to glycopeptides. Tolerance refers to persistence of nondividing 
viable organisms. In clinical isolates of $S$ aureus from patients with endocarditis or bacteremia, 7 of 15 MRSA isolates, but only 2 of 20 MSSA isolates, were tolerant as judged by time-kill experiments $(P=0.04)$ [55]. Glycopeptide tolerance is a real phenomenon in $S$ aureus, particularly among MRSA isolates, and may compromise glycopeptide therapy for serious $S$ aureus infection. These observations, together with the emergence of isolates with reduced susceptibility to glycopeptides, raise concern for continuing therapy with vancomycin as standard therapy for pneumonia or bacteremia caused by gram-positive cocci, especially in critically ill patients in the absence of rapid clinical response.

The significance of heteroresistant $S$ aureus strains remains to be analyzed. Heteroresistance refers to the heterogenous or differential expression of resistance within a colony. It was first noted with regard to methicillin-resistant staphylococci and has been more recently described for vancomycin/MRSA interaction. In heteroresistant populations, the majority of cells expresses resistance to low concentrations of vancomycin, and a minority of cells expresses resistance to much higher $(8$ $\mu \mathrm{g} / \mathrm{mL}$ ) concentrations [56].

The MRSA strains characterized by heterogeneous vancomycin resistance or hGISA have been well described $[13,14]$. Vancomycin heteroresistance is inducible by $\beta$-lactam therapy. A case-control study to define risk factors for heteroresistance identified: the time between admission and onset of bacteremia, admission to the intensive care unit, the prior use of vancomycin or $\beta$-lactams, and the isolation of methicillin-resistant staphylococci as significant risk factors by use of univariate analysis [57]. Patients with bacteremia due to staphylococci with heteroresistance to vancomycin had an overall mortality rate of $44 \%$, compared with a mortality rate of $10 \%$ among patients with staphylococcal bacteremia without vancomycin heteroresistance.

At present, routine laboratory detection of $S$ aureus with intermediate resistance and heteroresistance is difficult, and procedures are not yet standardized. Screening for vancomycin resistance is done by conventional methods or by the disk-agar method that uses vancomycin-salt agar to demonstrate satellitism around an aztreonam disk, leading to the determination of vancomycin MIC by Etest [57].

\section{VRE prevalence}

The NNIS system has reported that $25 \%$ of enterococci are vancomycin resistant [58]. Given the propensity of enterococci to develop multidrug resistance, the panel agreed that an institutional incidence of resistance of $>10 \%$ should be considered high. Patients with individual risk factors for VRE infection requiring empiric therapy in such institutions should receive therapy active against VRE $[45,49]$.

\section{Enterococcal resistance to antibiotics}

Compared with streptococci, enterococci are relatively resistant to penicillin, ampicillin, and the ureidopenicillins, with MICs of $1 \mu \mathrm{g} / \mathrm{mL}$ to $8 \mu \mathrm{g} / \mathrm{mL}$ and even higher for most E faecium. Enterococci are also inherently resistant to other antibiotics, including cephalosporins, the semisynthetic penicillinase-resistant penicillins, and have developed plasmid- and transposon-mediated resistance to tetracyclines, erythromycin, azithromycin, clarithromycin, chloramphenicol, trimethoprim, and clindamycin. Enterococcal resistance to aminoglycosides results from the ability of enterococci to block the uptake of the drug at the cell wall [60]. Consequently, aminoglycosides are only effective against enterococci when used in combination with cell-wall-active antibiotics. This combination treatment has been compromised, however, by the rapid spread of high-level aminoglycoside resistance among enterococci $(>2,000 \mu \mathrm{g} / \mathrm{mL})$. This degree of resistance predicts an absence of synergism between cell-wall-active agents and the aminoglycoside. The mechanism of high-level resistance is the result of a bifunctional enzyme, which is capable of inactivating different aminoglycosides [61].

\section{Genetics of vancomycin resistance in enterococci}

The propensity of $E$ faecalis to acquire multiple antibiotic-resistance traits is in part due to the existence of multiple mechanisms for exchanging genetic material (conjugation). The best-studied system of conjugation involves oligopeptides called pheromones and pheromone-responsive plasmids. Briefly, strains of $E$ faecalis secrete into the culture medium a variety of small peptide sex pheromones specific for different types of plasmids. When a bacterium containing a pheromone-responsive plasmid (the potential donor cell) comes into contact with its corresponding pheromone, there is transcription of the specific plasmid gene, resulting in the expression of a peptide called "aggregation substance" on the cell surface. When this bacterium subsequently collides with another $E$ faecalis, the aggregation substance interacts with a corresponding binding substance on the surface of the colliding cell, bonding the two bacterial cells together. Such bonding provides a physical channel for the transference of the pheromone-responsive plasmid from the donor bacterium to the colliding or recipient bacterium. Once the recipient cell has acquired the plasmid, the passage of the resistance trait is complete and synthesis of the corresponding sex pheromone is shut off. Other conjugation systems involving a broad range of plasmids have also been implicated in the transfer of resistance among species of enterococci and other gram-positive organisms such as streptococci and staphylococci.

An additional type of conjugation, which involves conjugative transposons, may explain the spread of resistance genes to many different species [62]. Conjugative transposons also encode the ability to bring about conjugation 
between different bacterial cells. They insert into the chromosome or into a plasmid of the new host. Other resistance genes, including those encoding resistance to erythromycin and kanamycin, are also found on conjugative transposons. There are reports of the $v a n B$ gene cluster within large conjugative chromosomal elements that appear similar, at least in function, to conjugative transposons [63]. One of the more frightening issues being contemplated with regard to vancomycin resistance in enterococci is the clinical consequence if and when these transferable vancomycin resistance operons make the genus jump from Enterococcus to organisms such as $S$ aureus (including MRSA) and Streptococcus pneumoniae. Transfer of VRE genes from the enterococci to other bacteria has already occurred in nature, and investigators have demonstrated the transferability of the vanA operon from Enterococcus into other species, including $S$ aureus, in the laboratory.

\section{Biochemistry of vancomycin resistance}

In vancomycin susceptible strains, the binding of vancomycin to D-alanine-D-alanine (D-ala) termini of peptidoglycan precursors prevents their cross-linking to adjacent strands. This significantly weakens the structural integrity of the cell wall and leads to osmotic lysis. Vancomycin resistance in enterococci is the result of the production of peptidoglycan precursors that terminate in D-ala-D-lactate instead of D-ala-D-ala, and these altered peptidoglycan precursors show greatly decreased binding affinity for glycopeptide antibiotics [64]. Two major, genetically distinct forms of acquired resistance, vanA and vanB, are located on transposable elements and respectively confer high-level resistance to both vancomycin and teicoplanin or to vancomycin alone [65].

The major risk factor for systemic infection with VRE is gastrointestinal colonization by VRE. One predisposing factor for gut colonization by VRE is the use of oral or parenteral antimicrobial agents with activity against anaerobes. In a recent study, oral or parenteral metronidazole, but not vancomycin, was correlated with VRE bacteremia $[66,67]$. This finding is of concern because metronidazole has recently been advocated over oral vancomycin as the drug of choice for antibiotic-induced colitis, with the reasoning that oral vancomycin would predispose to gut colonization with VRE.

\section{Duration of treatment}

How long should treatment be continued in patients initiated with empiric antibiotic therapy? In general, treatment should be continued and driven by culture and susceptibility data and should be stopped or changed based on these results.

Treatment should also be guided by patient response. Any treatment may be considered unsuccessful if there is a lack of clinical response in 72 hours, as indicated by the persistence of fever, high white blood cell count, and other signs of systemic infection such as persistent bacteremia. Persistent organ dysfunction, whether at the site of infection or not, may also be indicative of treatment failure. The status of therapy may be periodically assessed with cultures, particularly for infections in debilitated or immunocompromised patients where prolonged treatment ( $>7$ days) may be required. Such patients are at greatest risk of acquisition of resistance on therapy [68,69]. Resistance has occurred primarily in patients who did not have a foreign body (eg, a peritoneal dialysis catheter) removed. The panel believes that measurement of the rate of pathogen clearance from quantitative cultures should be explored as an index of treatment response.

Since oral formulations of $\beta$-lactams and linezolid are available, on-going patient evaluation should include consideration of oral therapy. This alternative may have the potential to reduce hospital LOS. Although there appears to be a reluctance to use oral therapy in seriously ill patients, the efficacy and bioavailability (100\%) of linezolid now make therapy possible in those patients who do not require hospitalization but otherwise require parenteral vancomycin therapy [70,71]. The panel recommends that conversion to oral therapy be considered in patients in whom discharge is eminent (within 48 hours) as long as they meet common criteria for oral drug administration (functioning gastrointestinal tract, absence of hypotension, absence of evidence of multiple organ dysfunction syndrome).

\section{Cost of newer therapies}

Acquisition costs for both linezolid and Q/D are substantially higher than acquisition costs for vancomycin. Per day of intravenous therapy, the cost of linezolid and Q/D are approximately $\$ 140$ and $\$ 320$, respectively [72]. Oral linezolid is slightly less expensive (approximately $\$ 106$ ) than the intravenous formulation per day of therapy. However, a significant proportion of the overall cost of treating a serious infection is incurred from the patient occupying a hospital bed [73]. Data collected from several studies of linezolid and $\mathrm{Q} / \mathrm{D}$ have determined that hospital length of stay is 1 to 2.5 days shorter with linezolid or Q/D compared with vancomycin, oxacillin, and cefazolin [74-76]. In addition, because there is no oral alternative to vancomycin other than linezolid, mean and median durations of intravenous treatment were significantly shorter $(P<.001)$ for patients treated with linezolid $(n=240)$ compared with patients treated with vancomycin $(n=220)$, with a mean difference of 5.6 days in the intent-to-treat sample [75]. The combined duration of intravenous and oral therapy for patients treated with linezolid was slightly longer compared with patients treated with vancomycin (13.1 days versus 11.8 days; $P=$ 0.06). Although the acquisition costs of linezolid and Q/D are higher than those for vancomycin, there may be other economic benefits to using these drugs such as fewer days in the hospital, and, in the case of linezolid, fewer days of 
intravenous therapy with its associated nursing and pharmacy costs.

\section{Conclusions}

Drug resistance among gram-positive pathogens is currently an endemic problem and is prevalent across antibiotic classes. The mechanisms of $\beta$-lactam resistance among gram-positive organisms are substantially different than those found in gram-negative organisms. This is also true for vancomycin-resistant organisms. The genetics and biochemistry of vancomycin resistance are central to determining the likelihood of continued spread of these organisms and the probability that this characteristic will be passed on to other organisms. With its propensity to acquire new traits, such as high-level gentamicin, penicillin, and vancomycin resistance, the enterococcus continues to create new therapeutic problems and dilemmas. Its ability to transfer plasmids to streptococci and staphylococci and the implications of a possible spread of penicillin and vancomycin resistance to these and other gram-positive species are also of concern. It is unclear whether a campaign to reduce vancomycin usage will be successful as a strategy to control the spread of vancomycin resistance. The availability of linezolid and Q/D offers new options for treatment of gram-positive infections. By instituting proper in-house control guidelines against infection, implementing methods for appropriate antibiotic usage, and developing models for reduction of resistance against these new agents, it is hoped that the utility of these new antibiotics will be extended.

We have focused on postsurgical infections and highlighted the decisions that need to be made in selecting appropriate antibiotics, routes of administration, duration of treatment, and assessment of efficacy of treatment. Empiric therapy should be initiated early in the course of infection in all seriously ill patients. The choice of therapy should flow from $\beta$-lactams to vancomycin to parenteral linezolid or Q/D. In patients in whom discharge is anticipated, and who are candidates for oral therapy, oral linezolid is an option for the treatment of resistant gram-positive infections. We emphasize an approach to provide antibiotic therapy that will cover the most virulent species, identified by risk factors, with subsequent coverage that will target culture-identified organisms. While many gaps remain in our knowledge, we hope that the decision tree presented here contributes to a logical treatment approach, one in which decisions are made after weighing both individual and institutional risks for colonization and resistance.

\section{Future directions}

The panel recommends that diligent institutional surveillance and resistance data be maintained so that treatment decisions can be made logically and problems of resistance can be identified early in development. Although we have recommended that an MRSA prevalence rate $>20 \%$ and VRE prevalence $>10 \%$ should be considered high in any institution, we recognize that research is needed to validate such cut-off points.

With the availability of newer agents, it has become possible to tailor therapy to the specific patient, the particular site of infection, and the specific pathogen. These options provide treatment alternatives that may prevent specific organ toxicities (eg, nephrotoxicity from vancomycin) or permit drug accumulation at an infection site. This may improve efficacy for that agent (eg, at pulmonary epithelial lining for the treatment of pneumonia or on the skin for cellulitis). Combination therapies are currently under investigation as are antibiotic cycling programs. Their relative success remains to be established. Exposure to antibiotics with differing mechanisms of action also implies a reduction in resistance selection pressure, a development that should be welcomed. Another area that could use additional research is the necessary duration of treatment for specific infections and evidence to support specific stopping criteria. It is possible that serial quantitative cultures, particularly for pulmonary infections, could prove to be useful in this context.

\section{Acknowledgments}

This work was supported in part by an unrestricted grant from Pharmacia Corp.

\section{References}

[1] Edmond MB, Wallace SE, McClish DK, et al. Nosocomial bloodstream infections in United States hospitals: a three-year analysis. Clin Infect Dis 1999;29:239-44.

[2] Richards MJ, Edwards JR, Culver DH, Gaynes RP. Nosocomial infections in combined medical-surgical intensive care units in the United States. Infect Control Hosp Epidemiol 2000;21:510-15.

[3] Jones RN, Low DE, Pfaller MA. Epidemiologic trends in nosocomial and community-acquired infections due to antibiotic-resistant Grampositive bacteria: the role of streptogramins and other newer compounds [in process citation]. Diagn Microbiol Infect Dis 1999;33: 101-12.

[4] Kollef MH, Ward S. The influence of mini-BAL cultures on patient outcomes: implications for the antibiotic management of ventilatorassociated pneumonia. Chest 1998;113:412-20.

[5] Montravers P, Gauzit R, Muller C, et al. Emergence of antibioticresistant bacteria in cases of peritonitis after intraabdominal surgery affects the efficacy of empirical antimicrobial therapy. Clin Infect Dis 1996;23:486-94.

[6] Chow JW, Fine MJ, Shlaes DM, et al. Enterobacter bacteremia: clinical features and emergence of antibiotic resistance during therapy. Ann Intern Med 1991;115:585-90.

[7] Romero-Vivas J, Rubio M, Fernandez C, Picazo JJ. Mortality associated with nosocomial bacteremia due to methicillin-resistant Staphylococcus aureus. Clin Infect Dis 1995;21:1417-23.

[8] Leibovici L, Shraga I, Drucker M, et al. The benefit of appropriate empirical antibiotic treatment in patients with bloodstream infection. J Intern Med 1998;244:379-86. 
[9] Hartman BJ, Tomasz A. Expression of methicillin resistance in heterogeneous strains of Staphylococcus aureus. Antimicrob Agents Chemother 1986;29:85-92.

[10] Hurlimann-Dalel RL, Ryffel C, Kayser FH, Berger-Bachi B. Survey of the methicillin resistance-associated genes mecA, mecR1-mecI, and femA-femB in clinical isolates of methicillin-resistant Staphylococcus aureus. Antimicrob Agents Chemother 1992;36:2617-21.

[11] Hiramatsu K, Hanaki H, Ino T, et al. Methicillin-resistant Staphylococcus aureus clinical strain with reduced vancomycin susceptibility. J Antimicrob Chemother 1997;40:135-6.

[12] Smith TL, Pearson ML, Wilcox KR, et al. Emergence of vancomycin resistance in Staphylococcus aureus. Glycopeptide-Intermediate Staphylococcus aureus Working Group. N Engl J Med 1999;340:493-501.

[13] Hiramatsu K, Aritaka N, Hanaki H, et al. Dissemination in Japanese hospitals of strains of Staphylococcus aureus heterogeneously resistant to vancomycin. Lancet 1997;350:1670-3.

[14] Ariza J, Pujol M, Cabo J, et al. Vancomycin in surgical infections due to methicillin-resistant Staphylococcus aureus with heterogeneous resistance to vancomycin. Lancet 1999;353:1587-8.

[15] Sieradzki K, Villari P, Tomasz A. Decreased susceptibilities to teicoplanin and vancomycin among coagulase-negative methicillin-resistant clinical isolates of staphylococci. Antimicrob Agents Chemother 1998;42:100-7.

[16] Donskey CJ, Chowdhry TK, Hecker MT, et al. Effect of antibiotic therapy on the density of vancomycin-resistant enterococci in the stool of colonized patients. N Engl J Med 2000;343:1925-32.

[17] Stephenson J. CDC campaign targets antimicrobial resistance in hospitals. JAMA 2002;287:2351-2.

[18] Pearson ML. Guideline for prevention of intravascular device-related infections. Hospital Infection Control Practices Advisory Committee. Infect Control Hosp Epidemiol 1996;17:438-73.

[19] Mangram AJ, Horan TC, Pearson ML, et al. Guideline for prevention of surgical site infection, 1999. Hospital Infection Control Practices Advisory Committee [see comments]. Infect Control Hosp Epidemiol 1999;20:250-278.

[20] Grossman RF, Fein A. Evidence-based assessment of diagnostic tests for ventilator-associated pneumonia. Executive summary. Chest 2000;117:177S-81S.

[21] Ibrahim EH, Sherman G, Ward S, et al. The influence of inadequate antimicrobial treatment of bloodstream infections on patient outcomes in the ICU setting. Chest 2000;118:146-55.

[22] Topeli A, Unal S, Akalin HE. Risk factors influencing clinical outcome in Staphylococcus aureus bacteraemia in a Turkish University Hospital. Int J Antimicrob Agents 2000;14:57-63.

[23] Malanoski GJ, Samore MH, Pefanis A, Karchmer AW. Staphylococcus aureus catheter-associated bacteremia. Minimal effective therapy and unusual infectious complications associated with arterial sheath catheters. Arch Intern Med 1995;155:1161-6.

[24] Mermel LA, Farr BM, Sherertz RJ, et al. Guidelines for the management of intravascular catheter-related infections. Clin Infect Dis 2001;32:1249-72.

[25] Panlilio AL, Culver DH, Gaynes RP, et al. Methicillin-resistant Staphylococcus aureus in U.S. hospitals, 1975-1991. Infect Control Hosp Epidemiol 1992;13:582-6.

[26] Fortun J, Navas E, Martinez-Beltran J, et al. Short-course therapy for right-side endocarditis due to Staphylococcus aureus in drug abusers: cloxacillin versus glycopeptides in combination with gentamicin. Clin Infect Dis 2001;33:120-5.

[27] Wood MJ. Comparative safety of teicoplanin and vancomycin. J Chemother 2000;12(suppl 5):21-5.

[28] Rybak MJ, Abate BJ, Kang SL, et al. Prospective evaluation of the effect of an aminoglycoside dosing regimen on rates of observed nephrotoxicity and ototoxicity. Antimicrob Agents Chemother 1999; 43:1549-55.

[29] Barriere JC, Berthaud N, Beyer D, et al. Recent developments in streptogramin research. Curr Pharm Des 1998;4:155-80.
[30] Lamb HM, Figgitt DP, Faulds D. Quinupristin/dalfopristin: a review of its use in the management of serious gram-positive infections. Drugs 1999;58:1061-97.

[31] Allington DR, Rivey MP. Quinupristin/dalfopristin: a therapeutic review. Clin Ther 2001;23:24-44.

[32] Shinabarger DL, Marotti KR, Murray RW, et al. Mechanism of action of oxazolidinones: effects of linezolid and eperezolid on translation reactions. Antimicrob Agents Chemother 1997;41:2132-6.

[33] Cercenado E, Garcia-Garrote F, Bouza E. In vitro activity of linezolid against multiply resistant Gram-positive clinical isolates. J Antimicrob Chemother 2001;47:77-81.

[34] Noskin GA, Siddiqui F, Stosor V, et al. In vitro activities of linezolid against important gram-positive bacterial pathogens including vancomycin-resistant enterococci. Antimicrob Agents Chemother 1999;43: 2059-62.

[35] Johnson AP, Warner M, Livermore DM. Activity of linezolid against multi-resistant gram-positive bacteria from diverse hospitals in the United Kingdom. J Antimicrob Chemother 2000;45:225-30.

[36] von Eiff C, Peters G. Comparative in-vitro activities of moxifloxacin, trovafloxacin, quinupristin/dalfopristin and linezolid against staphylococci. J Antimicrob Chemother 1999;43:569-73.

[37] Patel R, Rouse MS, Piper KE, Steckelberg JM. In vitro activity of linezolid against vancomycin-resistant enterococci, methicillin-resistant Staphylococcus aureus and penicillin-resistant Streptococcus pneumoniae. Diagn Microbiol Infect Dis 1999;34:119-22.

[38] Plouffe JF. Emerging therapies for serious gram-positive bacterial infections: a focus on linezolid. Clin Infect Dis 2000;31(suppl 4): 144-9.

[39] Rubinstein E, Cammarata S, Oliphant T, Wunderink R. Linezolid [PNU-100766] versus vancomycin in the treatment of hospitalized patients with nosocomial pneumonia: a randomized, double-blind, multicenter study. Clin Infect Dis 2001;32:402-12.

[40] Gerson SL, Kaplan SL, Bruss JB, et al. Hematologic effects of linezolid: summary of clinical experience. Antimicrob Agents Chemother 2002;46:2723-6.

[41] Levine DP, Fromm BS, Reddy BR. Slow response to vancomycin or vancomycin plus rifampin in methicillin-resistant Staphylococcus aureus endocarditis. Ann Intern Med 1991;115:674-80.

[42] Small PM, Chambers HF. Vancomycin for Staphylococcus aureus endocarditis in intravenous drug users. Antimicrob Agents Chemother 1990;34:1227-31.

[43] Khatib R, Riederer KM, Held M, Aljundi H. Protracted and recurrent methicillin-resistant Staphylococcus aureus bacteremia despite defervescence with vancomycin therapy. Scand J Infect Dis 1995;27:52932.

[44] Jones SM, Morgan M, Humphrey TJ, Lappin-Scott H. Effect of vancomycin and rifampicin on meticillin-resistant Staphylococcus aureus biofilms. Lancet 2001;357:40-1.

[45] Barie PS. Antibiotic-resistant gram-positive cocci: implications for surgical practice. World J Surg 1998;22:118-26.

[46] Bodi M, Ardanuy C, Rello J. Impact of Gram-positive resistance on outcome of nosocomial pneumonia. Crit Care Med 2001;29:N82-6.

[47] Gonzalez C, Rubio M, Romero-Vivas J, et al. Bacteremic pneumonia due to Staphylococcus aureus: a comparison of disease caused by methicillin-resistant and methicillin-susceptible organisms. Clin Infect Dis 1999;29:1171-7.

[48] Moise PA, Schentag JJ. Vancomycin treatment failures in Staphylococcus aureus lower respiratory tract infections. Int $\mathrm{J}$ Antimicrob Agents 2000;16(suppl 1):S31-4.

[49] Hershow RC, Khayr WF, Smith NL. A comparison of clinical virulence of nosocomially acquired methicillin-resistant and methicillinsensitive Staphylococcus aureus infections in a university hospital. Infect Control Hosp Epidemiol 1992;13:587-93.

[50] Marty L, Flahault A, Suarez B, et al. Resistance to methicillin and 
virulence of Staphylococcus aureus strains in bacteriemic cancer patients. Intens Care Med 1993;19:285-9.

[51] Cosgrove SE, Sakoulas G, Perencevich EN, et al. Comparison of mortality associated with methicillin-resistant and methicillin-susceptible Staphylococcus aureus bacteremia: a meta-analysis. Clin Infect Dis 2003;36:53-9.

[52] Conterno LO, Wey SB, Castelo A. Risk factors for mortality in Staphylococcus aureus bacteremia. Infect Control Hosp Epidemiol 1998;19:32-7.

[53] Abramson MA, Sexton DJ. Nosocomial methicillin-resistant and methicillin-susceptible Staphylococcus aureus primary bacteremia: at what costs? Infect Control Hosp Epidemiol 1999;20:408-11.

[54] Rubin RJ, Harrington CA, Poon A, et al. The economic impact of Staphylococcus aureus infection in New York City hospitals. Emerg Infect Dis 1999;5:9-17.

[55] May J, Shannon K, King A, French G. Glycopeptide tolerance in Staphylococcus aureus. J Antimicrob Chemother 1998;42:189-97.

[56] Ryffel C, Strassle A, Kayser FH, Berger-Bachi B. Mechanisms of heteroresistance in methicillin-resistant Staphylococcus aureus. Antimicrob Agents Chemother 1994;38:724-8.

[57] Wong SS, Ho PL, Woo PC, Yuen KY. Bacteremia caused by staphylococci with inducible vancomycin heteroresistance. Clin Infect Dis 1999;29:760-7.

[58] Solomkin JS, Meakins JL, Allo MD, et al. Antibiotic trials in intraabdominal infections. A critical evaluation of study design and outcome reporting. Ann Surg 1984;200:29-39.

[59] Najjar A, Murray BE. Failure to demonstrate a consistent in vitro bactericidal effect of trimethoprim-sulfamethoxazole against enterococci. Antimicrob Agents Chemother 1987;31:808-10.

[60] Moellering RC, Weinberg AN. Studies on antibiotic syngerism against enterococci. II. Effect of various antibiotics on the uptake of 14 C-labeled streptomycin by enterococci. J Clin Invest 1971;50: 2580-4.

[61] Ferretti JJ, Gilmore KS, Courvalin P. Nucleotide sequence analysis of the gene specifying the bifunctional 629 -aminoglycoside acetyltransferase 2"-aminoglycoside phosphotransferase enzyme in Streptococcus faecalis and identification and cloning of gene regions specifying the two activities. J Bacteriol 1986;167:631-8.

[62] Scott JR, Churchward GG. Conjugative transposition. Annu Rev Microbiol 1995;49:367-97.

[63] Garnier F, Taourit S, Glaser P, et al. Characterization of transposon Tn1549, conferring VanB-type resistance in Enterococcus spp. Microbiology 2000;146:1481-9.

[64] Lessard IA, Walsh CT. Mutational analysis of active-site residues of the enterococcal D-ala-D-Ala dipeptidase VanX and comparison with Escherichia coli D-ala-D-Ala ligase and D-ala-D-Ala carboxypeptidase VanY. Chem Biol 1999;6:177-87.

[65] Woodford N. Glycopeptide-resistant enterococci: a decade of experience. J Med Microbiol 1998;47:849-62.

[66] Lucas GM, Lechtzin N, Puryear DW, et al. Vancomycin-resistant and vancomycin-susceptible enterococcal bacteremia: comparison of clinical features and outcomes. Clin Infect Dis 1998;26:1127-33.

[67] Lautenbach E, Bilker WB, Brennan PJ. Enterococcal bacteremia: risk factors for vancomycin resistance and predictors of mortality. Infect Control Hosp Epidemiol 1999;20:318-23.

[68] Pai MP, Rodvold KA, Schreckenberger PC, et al. Risk factors associated with the development of infection with linezolid- and vancomycin-resistant Enterococcus faecium. Clin Infect Dis 2002;35:1269-72.

[69] Tsiodras S, Gold HS, Sakoulas G, et al. Linezolid resistance in a clinical isolate of Staphylococcus aureus. Lancet 2001;358:207-8.

[70] Norrby R. Linezolid-a review of the first oxazolidinone. Expert Opin Pharmacother 2001;2:293-302.

[71] Perry CM, Jarvis B. Linezolid: a review of its use in the management of serious gram-positive infections. Drugs 2001;61:525-51.
[72] Rehm SJ. Two new treatment options for infections due to drugresistant gram-positive cocci. Cleve Clin J Med 2002;69:397-13.

[73] Nathwani D, Malek M. Cost considerations in the evaluation of new therapies for gram-positive bacteria. Int J Antimicrob Agents 1999; 13:71-8.

[74] Carbon C. Costs of treating infections caused by methicillin-resistant staphylococci and vancomycin-resistant enterococci. J Antimicrob Chemother 1999;44(suppl A):31-6.

[75] Li Z, Willke RJ, Pinto LA, et al. Comparison of length of hospital stay for patients with known or suspected methicillin-resistant Staphylococcus species infections treated with linezolid or vancomycin: a randomized, multicenter trial. Pharmacotherapy 2001;21:263-74.

[76] Willke RJ, Glick HA, Li JZ, Rittenhouse BE. Effects of linezolid on hospital length of stay compared with vancomycin in treatment of methicillin-resistant Staphylococcus infections. An application of multivariate survival analysis. Int $\mathrm{J}$ Technol Assess Health Care 2002; 18:540-54.

[77] Tentolouris N, Jude EB, Smirnof I, et al. Methicillin-resistant Staphylococcus aureus: an increasing problem in a diabetic foot clinic. Diabet Med 1999;16:767-71.

[78] Trividic M, Gauthier ML, Sparsa A, et al. Methi-resistant Staphylococcus aureus in dermatological practice: origin, risk factors and outcome [translation]. Ann Dermatol Venereol 2002;129:27-9.

[79] Cars O. Colonisation and infection with resistant gram-positive cocci. Epidemiology and risk factors. Drugs 1997;54(suppl 6):4-10.

[80] Rao GG. Risk factors for the spread of antibiotic-resistant bacteria. Drugs 1998;55:323-30.

[81] Fierobe L, Decre D, Muller C, et al. Methicillin-resistant Staphylococcus aureus as a causative agent of postoperative intra-abdominal infection: relation to nasal colonization. Clin Infect Dis 1999;29: 1231-8.

[82] Rojo D, Pinedo A, Clavijo E, et al. Analysis of risk factors associated with nosocomial bacteraemias. J Hosp Infect 1999;42:135-41.

[83] Jensen AG, Wachmann CH, Poulsen KB, et al. Risk factors for hospital-acquired Staphylococcus aureus bacteremia. Arch Intern Med 1999;159:1437-44.

[84] Rezende NA, Blumberg HM, Metzger BS, et al. Risk factors for methicillin-resistance among patients with Staphylococcus aureus bacteremia at the time of hospital admission. Am J Med Sci 2002; 323:117-23.

[85] McClelland RS, Fowler VG, Sanders LL, et al. Staphylococcus aureus bacteremia among elderly vs younger adult patients: comparison of clinical features and mortality. Arch Intern Med 1999;159:1244-7.

[86] Mylotte JM, Tayara A. Staphylococcus aureus bacteremia: predictors of 30-day mortality in a large cohort. Clin Infect Dis 2000;31:1170-4.

[87] Martone WJ. Spread of vancomycin-resistant enterococci: why did it happen in the United States? Infect Control Hosp Epidemiol 1998;19: 539-45.

[88] Bignardi GE, Askew C. Workload may be related to the spread of MRSA and other infections. J Hosp Infect 2000;45:78-80.

[89] Dahms RA, Johnson EM, Statz CL, et al. Third-generation cephalosporins and vancomycin as risk factors for postoperative vancomycinresistant enterococcus infection. Arch Surg 1998;133:1343-6.

[90] Gin AS, Zhanel GG. Vancomycin-resistant enterococci. Ann Pharmacother 1996;30:615-24.

[91] Byers KE, Anglim AM, Anneski CJ, et al. A hospital epidemic of vancomycin-resistant Enterococcus: risk factors and control. Infect Control Hosp Epidemiol 2001;22:140-7.

[92] Dan M, Poch F, Leibson L, et al. Rectal colonization with vancomycin-resistant enterococci among high-risk patients in an Israeli hospital. J Hosp Infect 1999;43:231-8.

[93] Bhorade SM, Christenson J, Pohlman AS, et al. The incidence of and clinical variables associated with vancomycin-resistant enterococcal colonization in mechanically ventilated patients. Chest 1999;115: 1085-91. 
[94] Stosor V, Peterson LR, Postelnick M, Noskin GA. Enterococcus faecium bacteremia: does vancomycin resistance make a difference? Arch Intern Med 1998;158:522-7.

[95] Linden PK, Miller CB. Vancomycin-resistant enterococci: the clinical effect of a common nosocomial pathogen. Diagn Microbiol Infect Dis 1999;33:113-20.

[96] Armstrong-Evans M, Litt M, McArthur MA, et al. Control of transmission of vancomycin-resistant Enterococcus faecium in a longterm-care facility. Infect Control Hosp Epidemiol 1999;20:312-17.
[97] Strausbaugh LJ, Crossley KB, Nurse BA, Thrupp LD. Antimicrobial resistance in long-term-care facilities. Infect Control Hosp Epidemiol 1996;17:129-40.

[98] Bonten MJ, Slaughter S, Ambergen AW, et al. The role of "colonization pressure" in the spread of vancomycin-resistant enterococci: an important infection control variable. Arch Intern Med 1998;158:1127-32.

[99] Bonten MJ, Slaughter S, Hayden MK, et al. External sources of vancomycin-resistant enterococci for intensive care units. Crit Care Med 1998;26:2001-4. 